\title{
LAYOUT AND OPERATION OF DISTRIBUTION CENTRES FOR PERISHABLES
}

\author{
Rob A.C.M. Broekmeulen, ATO-DLO, NL-Wageningen
}

The efficiency of a distribution centre plays a crucial role in the marketing of perishables. Perishables like dairy products, meat, vegetables and fruits suffer from quality loss due to aging and breakdown. Quality loss can be reduced by adequate storage conditions like relative humidity and temperature, minimal handling and avoiding of product interactions like odour and hormone transmission. Most perishables from agricultural origin have strong seasonal fluctuations in production and demand. The absence of an uniform article coding system makes the operation of the distribution centre even more complex.

A distribution centre for perishables has to deal with peak stock levels and product interactions. The aim is to maximise flexibility towards the customers and to minimise quality loss during the distribution process at the same time. This has to be achieved with minimal cost using the available resources.

We have developed a multi-stage hierarchical decision approach that is capable of handling the complexity of the overall problem. The iterative decision process consists of the following steps:

1 Facility design and technology selection.

2 Item allocation.

First, product groups are assigned to storage type clusters like cold stores. This is done with multiply goal programming and quality change models. Second, the individual articles are assigned to storage locations like racks and shelves. We use a local search technique to solve a time-dependent version of the bin packing problem.

3 Order picker routing with respect to stable customer pallets.

The process is integrated in a PC-based decision support system. The system generates several good altematives in a relative short time.

We present the application of the developed planning approach and decision models to a distribution centre of vegetables and fruits

Keywords: decision support system, distribution centres, hierarchical planning, local search techniques, logistics, operations management, perishables. 